\title{
Inferior Mesenteric Artery Aneurysm: Case Report and Literature Review
}

\author{
Seiji Edogawa, MD, ${ }^{1}$ Takashi Shibuya, ${ }^{1}$ Kimihiro Kurose, ${ }^{1}$ Kazuki Sasaki, ${ }^{2}$ and Hirohiko Tomita ${ }^{2}$
}

\begin{abstract}
An inferior mesenteric artery (IMA) aneurysm is the rarest among visceral artery aneurysms. A 69-yearold man was referred to our hospital with an asymptomatic IMA aneurysm associated with occlusion of the superior mesenteric artery (SMA) and celiac artery (CA). After revascularization of the SMA with an 8-mm expanded polytetrafluoroethylene (ePTFE) graft, the aneurysm was resected, and the IMA was reconstructed. The "jet disorder" phenomenon has been thought to cause an IMA aneurysm in the case of CA and SMA obstruction. We consider it better to revascularize not only an IMA but also an SMA or CA for preventing that phenomenon.
\end{abstract}

Keywords: inferior mesenteric artery, aneurysm, visceral artery aneurysm

\section{INTRODUCTION}

A $\mathrm{n}$ inferior mesenteric artery (IMA) aneurysm is a less common type of a visceral artery aneurysm. We report a case of an IMA aneurysm associated with occlusion of the superior mesenteric artery (SMA) and celiac artery (CA), which is so rare that only 20 reports exist worldwide, as far as we know. ${ }^{1-6}$

\section{Case Report}

The patient was a 69-year-old man who was referred to our hospital with an asymptomatic IMA aneurysm, which had been discovered at his primary hospital during treatment of tuberculous pleuritis. He was a heavy

${ }^{1}$ Division of Vascular Surgery, Department of Cardiovascular Surgery, Osaka Medical Center for Cancer and Cardiovascular Diseases, Osaka, Japan

${ }^{2}$ Department of Pathology, Osaka Medical Center for Cancer and Cardiovascular Diseases, Osaka, Japan

Received: October 1, 2012; Accepted: January 11, 2013 Corresponding author: Seiji Edogawa, MD. Division of Surgery, Jieitai Maizuru Hospital, 1537-1 Sengenji, Maizuru, Kyoto 625-0026, Japan

Tel: +81-7-7362-2273, Fax: +81-7-7364-5125

E-mail: seiji_edogawa_1014@yahoo.co.jp smoker and suffered from hypertension. He did not have any other risk factors related to atherosclerosis. He did not suffer from abdominal angina.

On physical examination, there was a bruit around the umbilicus at the strongest, but there was no palpable abdominal mass or thrill.

The abdominal computed tomography (CT) angiograms revealed a saccular IMA aneurysm located at the root of IMA branching from the abdominal aorta with a maximum diameter of $2.8 \mathrm{~cm}$ (Fig. 1a). There was no intraluminal thrombus. Both SMA and CA were occluded at the roots (Fig. 1b). Both branches of IMA and marginal arteries, supplying the entire intra-abdominal organs, were tortuous and dilated.

Complete occlusion of the SMA and CA was also demonstrated by an abdominal ultrasound examination.

The patient underwent midline laparotomy with a transperitoneal approach to the abdominal aorta and IMA aneurysm. There were no bowel adhesions or ascites. We faced dilated and tortuous marginal arteries in the mesenterium. First, to minimalize the intestinal ischemia during the operation, we constructed an end-to-side bypass from the infrarenal aorta to the SMA with an 8-mm expanded polytetrafluoroethylene (ePTFE) graft (Fig. 2). Then, the IMA aneurysm was completely resected. The normal proximal and distal ends of the IMA were 
(a)

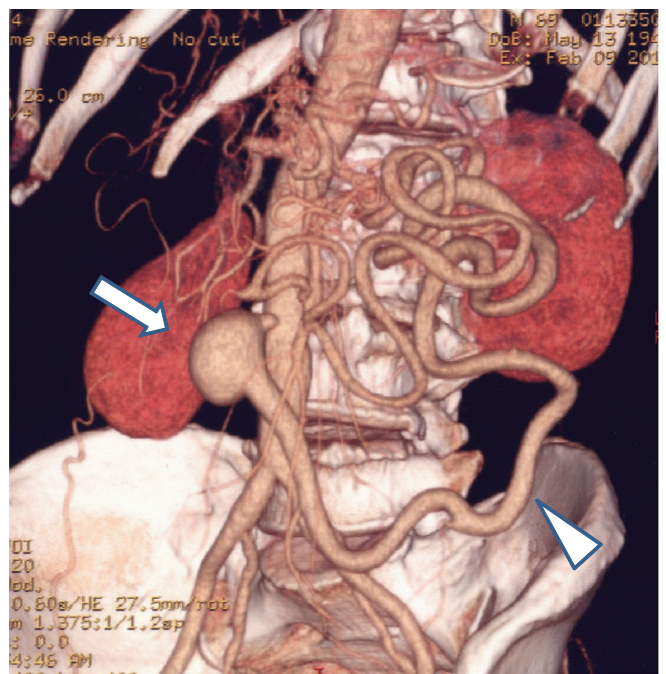

(b)

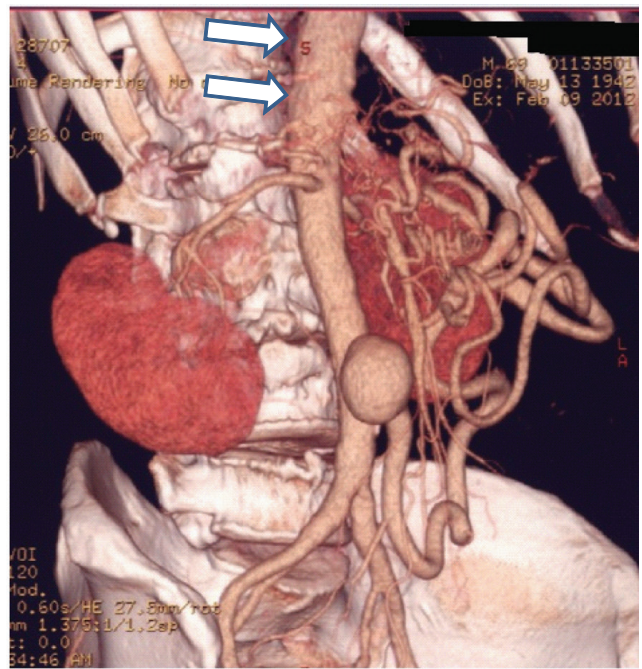

Fig. 1 (a) An abdominal CT angiogram displaying an IMA aneurysm (arrow), and a large dilated marginal artery (arrow head). (b) Arrows indicate occlusion of the CA and SMA.

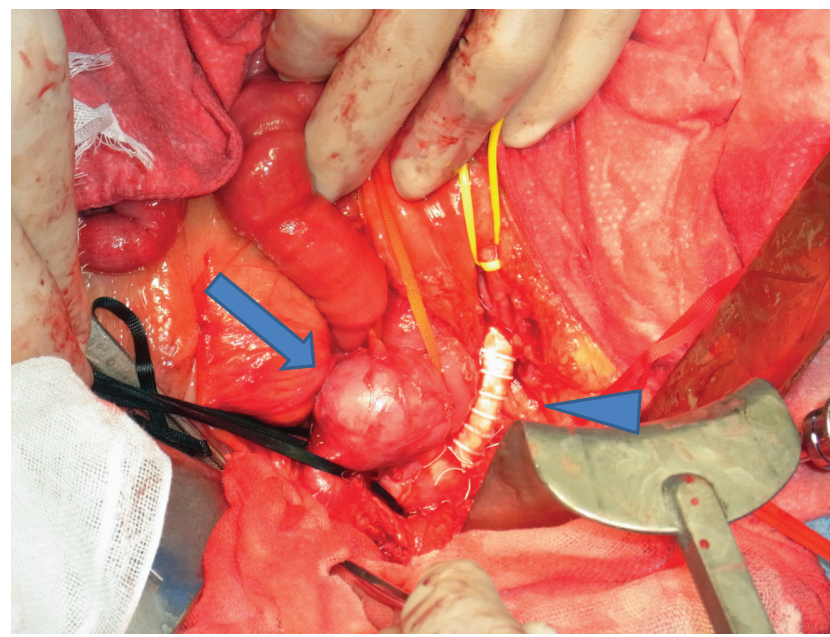

Fig. 2 Intraoperative view of the aorta, IMA aneurysm (arrow) and Aorta-SMA bypass (arrow head).

mobilized and reconnected in an end-to-end manner to re-establish blood flow. Finally, the retroperitoneum was closed completely so that the ePTFE graft was kept away from intestine.

Postoperative CT angiograms showede patency of the graft and IMA, and we confirmed a regression of the marginal artery (Fig. 3). Histology showed atherosclerotic disease without calcification (Fig. 4). There are findings of thickening of the intima, accompanied by atheromatous degeneration. Inflammatory cell infiltration is not remarkable, and there is no evidence of tuberculosis. The patient had an uneventful postoperative course and no symptoms of intestinal ischemia.

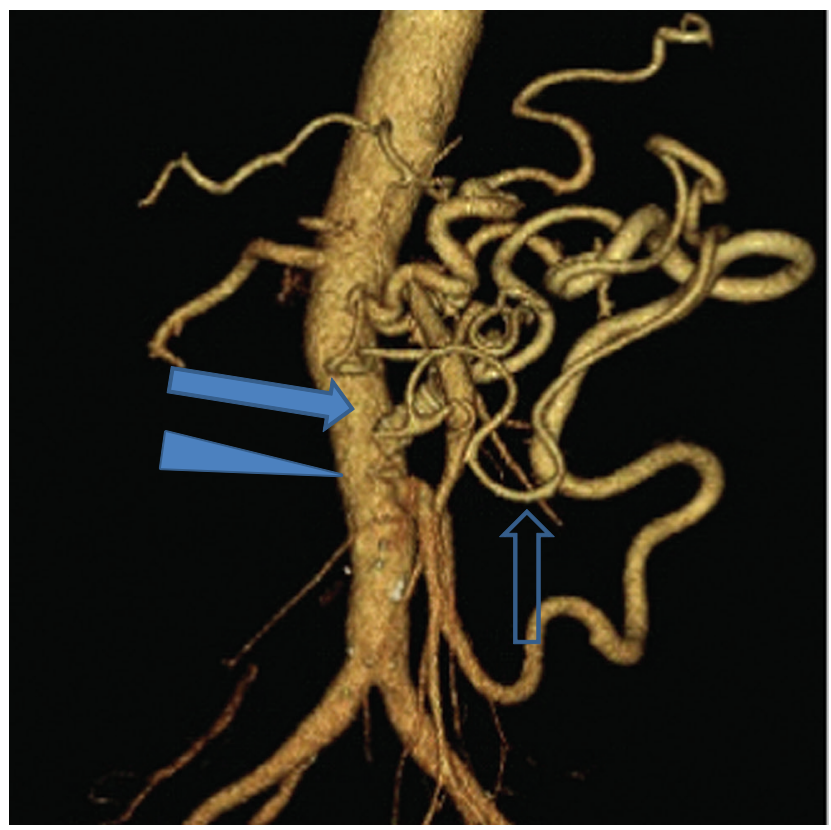

Fig. 3 Postoperative CT angiogram showing an aorta-SMA bypass (arrow), new anastomosis of IMA (arrow head) and regression of the marginal artery (open arrow).

\section{Discussion}

Reports of IMA aneurysm are increasing as diagnostic facility improves, however, IMA aneurysm is still the rarest among all visceral artery aneurysms. Distribution of aneurysms of the splanchnic vessels includes involvement of the splenic artery (60\%), hepatic artery (20\%), SMA (6\%), CA (4\%), gastric and gastroepiploic arteries (4\%), jejunal, ileal, and colic arteries (3\%), pancreatic 


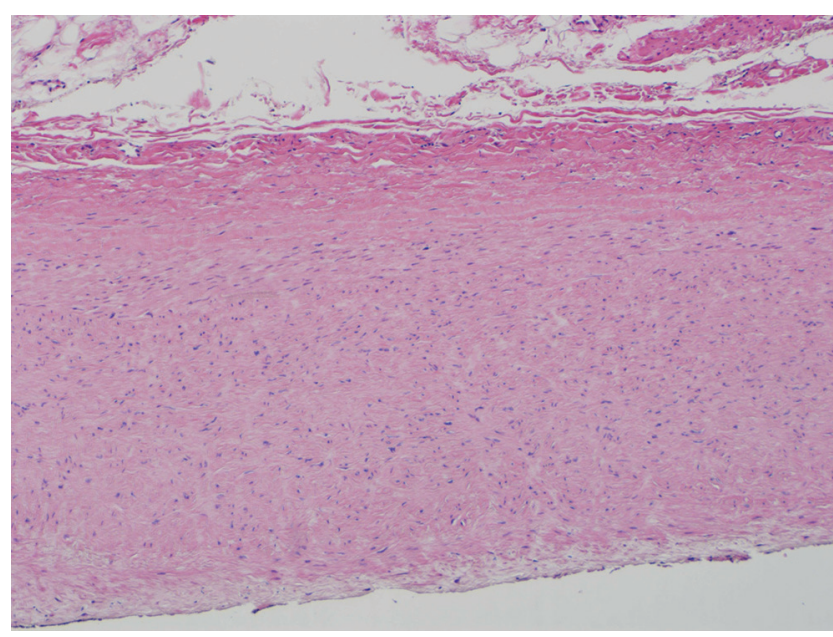

Fig. 4 Enlarged photograph of the IMA aneurysm wall $(\times 100)$.

duodenal and pancreatic arteries $(2 \%)$, gastroduodenal artery $(1.5 \%)$, and IMA, less than $\left.1 \% .{ }^{7}\right)$

We found 54 cases of IMA aneurysms including our own case by PubMed and Japan Medical Abstracts Society from 1861 to $2012 . .^{1-6,8-10)}$ The age of patients ranged between 9 and 84 years; there were 43 men and 11 women.

Atherosclerotic disease was the most common cause of IMA aneurysm, accounting for 22 cases, including our case. Other causes were mycotic, polyarteritis nodosa, a dissecting hematoma, Takayasu's disease, a false iatrogenic postoperative aneurysm, aortitis, segmental mediolytic arteritis, tuberculous, Behcet disease, and neurofibromatosis.

Most IMA aneurysms are localized in proximal trunk of IMA and close to half cases including our case show no symptoms. Symptoms include asymptomatic pulsatile/ expansile abdominal mass, abdominal pain, low back pain, and collapse or hemorrhagic shock due to rupture.

Including our case, there were 21 cases of IMA aneurysms associated with occlusion of the SMA and CA. ${ }^{1-6)}$ In these cases, atherosclerotic disease was the most common cause, which accounted for 12 cases including our case.

Other causes were Takayasu's disease, aortitis, Behcet disease, neurofibromatosis. In these cases, splanchnic blood flow was supplied only through the tortuous marginal arteries originating from the IMA. As mentioned by several authors, the greatly increased and possibly turbulent blood flow might create dilatation of IMA and small collateral arteries, because of the "jet disorder" phenomenon. ${ }^{2,4,5)}$ As a result, the marginal arteries were also greatly increased in size, probably reflecting sufficient collateralization due to chronic occlusion of SMA and CA. Similarly, we confirmed a dilated and tortuous marginal artery in the mesenterium.

The frequency of IMA aneurysmal rupture is yet to be known because of its rareness. According to the previously reported cases, we found 11 rupture cases of IMA aneurysm and 4 of which resulted in death. $., 3,4,6)$ Once IMA aneurysm ruptures, prognosis is poor just as another ruptured visceral aneurysm. Thus, surgical treatments seem to be the best way to prevent death from rupture. We suggest that if an IMA aneurysm is greater than $2 \mathrm{~cm}$ at the proximal or $1 \mathrm{~cm}$ at the distal of the IMA, operative intervention should be performed because of an increased risk of rupture. Uno, et al. introduced that a case of aneurysm at the distal site of the IMA was performed TAE. ${ }^{3)}$ This case resulted in death from rupture.

Surgical treatment should be selected on the basis of the status of the intestinal circulation. If both CA and SMA are patent, the IMA aneurysm can be treated simply by resection or ligation. However, if the IMA is the main tract of the entire intra-abdominal organ's blood flow such as in our case, revascularization of the aneurysm using bypass grafting or direct reimplantation is necessary.

It is best to revascularize both the CA and SMA whenever feasible. We think that revascularization of the SMA or CA has three significant points. First, it may be able to prevent recurrence of IMA aneurysm by suppressing the "jet disorder" phenomenon. Secondly, it can minimize intra-abdominal organ ischemia during an operation. Finally, it also prevents intra-abdominal organ ischemia after surgery by reducing the risk of later occlusion of IMA. There have been some reports of revascularization of SMA and IMA. Tsukioka, et al. reported that they confirmed regression of the middle colic artery in the postoperative $\mathrm{CT}$ angiogram after reconstruction of the SMA and IMA.5)

Similarly, we confirmed regression of the marginal artery in the postoperative CT angiogram. We intend to follow up for some time in the future. We selected an ePTFE graft between the SMA and the abdominal aorta instead of an autologous vein graft, because we thought that the prosthetic graft was more resistant to kinking by movement of the mesenterium. The use of an artificial graft is a risk factor of graft infection, but the graft was completely covered with retroperitoneum to keep it away from the intestine. Therefore, the risk of graft infection is very low. We think that, as well as artificial graft replacement of the abdominal aortic aneurysm, the use of an artificial graft was acceptable. 


\section{Conclusion}

We experienced a case of an IMA aneurysm associated with the occlusion of an SMA and CA. The addition of revascularization of the CA or SMA may prevent recurrence of the IMA aneurysm by suppressing the "jet disorder" phenomenon. We performed revascularization of both the IMA and SMA.

The postoperative CT angiogram showed regression of the marginal artery.

\section{Disclosure Statement}

The authors have no financial conflicts of interest to disclose concerning the presentation.

\section{REFERENCES}

1) Davidovic LB, Vasic DM, Colic MI. Inferior mesenteric artery aneurysm: case report and review of the literature. Asian J Surg 2003; 26: 176-9.

2) Mandeville KL, Bicknell $C$, Narula $S$, et al. Inferior mesenteric artery aneurysm with occlusion of the superior mesenteric artery, coeliac trunk and right renal artery. Eur J Vasc Endovasc Surg 2008; 35: 312-3. Epub 2007 Oct 29.
3) Uno Y, Kobayashi K, Ogiso S, et al. A case of inferior mesenteric two year after sigmoidectomy. Jpn J Gastroenterol Surg 2008; 41: 1735-40.

4) Pérez-Vallecillos P, Conde-Muíño R, Segura-Jiménez I, et al. Acute retroperitoneal bleeding due to inferior mesenteric artery aneurysm: case report. BMC Gastroenterol 2010; 10: 59.

5) Tsukioka K, Nobara H, Nishimura K. A case of inferior mesenteric artery aneurysm with an occlusive disease in superior mesenteric artery and the celiac artery. Ann Vasc Dis 2010; 3:160-3.

6) Werth S, Rodionov RN, Hinterseher I, et al. Rupture prone aneurysm of the inferior mesenteric artery complicated by mid aortic syndrome with occluded celiac and superior mesenteric arteries. VASA 2011; 40: 73-7.

7) Messina LM, Shanley CJ. Visceral artery aneurysms. Surg Clin North Am 1997; 77: 425-42. Epub 1997 April 1.

8) Momin AA, Chaubal NG, Saifi SG, et al. Sonographic diagnosis of inferior mesenteric artery aneurysm and marginal artery of Drummond. J Clin Ultrasound 2008; 36: 42-4.

9) Clark CE, Monzon JR, Gifford S, et al. Inferior mesenteric artery aneurysm in the setting of chronic colonic vascular ectasia. Ann Vasc Surg 2011; 25: 267. e7-9. Epub 2010 Oct 6.

10) Okamoto M, Tanaka M. A Case of Solitary Inferior Mesenteric Artery Aneurysm. Jpn J Cardiovasc Surg 2012; 41: 121-3. 